\title{
Razones, retos y aportaciones del programa de la especialidad de Medicina Familiar y Comunitaria en España
}

\author{
Razões, desafios e contribuições do programa da especialidade de Medicina de Família e Comunidade na Espanha \\ Reasons, challenges and contributions of the specialty training programme in family and community medicine in Spain
}

Verónica Casado Vicente. Centro de Salud Docente y Universitario Parquesol. Valladolid, Castilla y León, España. vcasadovicente@gmail.com Lourdes Luzón Oliver. Secretaria Municipal de Saúde do Rio de Janeiro (SMSRJ). Rio de Janeiro, RJ, Brasil. lourdesluzon@gmail.com (Autora correspondiente)

\section{Resumen}

El sistema de formación especializada en medicina mediante residencia ha sido indudablemente, en estos 30 años, uno de los motores que ha contribuido a elevar de forma homogénea la calidad asistencial y la seguridad de los pacientes, alcanzando de forma eficiente importantes resultados en salud en España, en relación a los países de nuestro entorno. La especialidad de Medicina Familiar y Comunitaria (MFyC) es una de las 44 especialidades médicas vía residencia. Se desarrolla en 97 Unidades Docentes (UUDD) que cuentan con 887 Centros de Salud y 212 hospitales acreditados y forma en torno a 1800 residentes/año. El programa de la especialidad actualmente vigente, de 4 años, es un programa orientado por competencias donde la flexibilidad, la pluripotencialidad, el eje tutor-residente y la evaluación formativa son sus características claves. Nace de la firme voluntad de actualizar y consolidar el cuerpo doctrinal y los valores de la MFyC y de un profundo compromiso con la formación y con el Sistema Nacional de Salud. Se sustenta en la evidencia de que un sistema sanitario que pretenda ser equitativo, efectivo, eficiente y viable ha de contar con una Atención Primaria potente y resolutiva, la cual debe ser proporcionada por médicos bien formados y entrenados en su práctica. Por todo ello, es clave que los Ministerios (y otros organismos responsables de los sistemas sanitario y educativo de los países) consideren como objetivo imprescindible valorizar, con financiación adecuada y "empowerment", la Atención Primaria como función central del sistema sanitario, y la MFyC como disciplina, especialidad y profesión sanitaria.

\section{Resumo}

0 sistema de formação especializada em medicina mediante residência tem sido, sem dúvida, nestes 30 anos, um dos elementos que contribuíram para aumentar a homogeneidade da qualidade assistencial e a segurança dos pacientes, atingindo eficientemente importantes resultados na saúde espanhola, com relação aos países vizinhos. A especialidade de Medicina de Família e Comunidade (MFC) é uma das 44 especialidades médicas existentes por meio de residência. É desenvolvida em 97 Unidades Docentes (UUDD) que contam com 887 Centros de Saúde e com 212 hospitais credenciados que formam cerca de 1800 residentes/ano. 0 programa da especialidade, que atualmente é de quatro anos, é orientado por competências em que a flexibilidade, o pluralismo na potencialidade, 0 eixo preceptor-residente, e a avaliação, são características principais. 0 programa nasce da vontade de atualizar e consolidar as doutrinas e os valores da MFC e de um profundo compromisso com a formação e com o Sistema Nacional de Saúde. É sustentado na evidência de que um sistema de saúde para ser equitativo, eficiente e viável precisa contar com uma Atenção Primária contundente e resolutiva, que deve vir com médicos bem formados e treinados. Por isso, é fundamental que os ministérios e os órgãos responsáveis pelos sistemas de saúde e educação dos diferentes países, levem em conta como objetivo imprescindível, a valorização, com um financiamento adequado e 0 empowerment necessário, da Atenção Primária como eixo central do sistema de saúde, e a MFC como disciplina, especialidade e profissão para a área da saúde.

\section{Palabras clave:}

Medicina Familiar y Comunitaria Internado y Residencia

Educación Médica

Educación Basada en Competencias

Reforma de la Atención de Salud

\section{Palavras-chave:}

Medicina de Família e Comunidade Internato e Residência Educação Médica Educação Baseada em Competências Reforma dos Serviços de Saúde 
Abstract

In the past 30 years, specialty training in medicine, by means of residency programmes, has undoubtedly been one of the instruments that helped raise care quality and patient safety homogenously across the country, efficiently achieving important health outcomes in Spain in relation to surrounding countries. The specialty of Family and Community Medicine (FCM) is one of 44 medical specialties which require a residency training programme. It is developed in 97 teaching units that count on 887 health centres and 212 accredited hospitals, and train approximately 1800 residents per year. Currently, the specialty programme in FCM lasts four years and it is a competency-oriented programme whose key elements encompass flexibility, pluripotency, a resident-preceptor axe, and formative assessment. It originates from the firm will to update and consolidate the doctrines and values of FCM, as well as from a deep commitment to education and the National Health System. It is based on the evidence that an equitable, efficient and viable health care system must rely on a forceful and resolute Primary Care, which should provide well-prepared and trained physicians. Therefore, it is critical that the Ministries (and other organisations responsible for the health and educational systems of different countries) regard the provision of adequate funding and empowerment as an essential objective, so that primary care services can play a central role within the Health System and FCM can be seen as a discipline, specialty and health profession.

"Si creemos en algo, si tenemos en nuestro interior suficiente energía, suficiente pasión y ganas de vivir, podemos encontrar en los recursos que nos ofrece el mundo actual los medios necesarios para hacer realidad nuestros sueños."

Amin Malouf

El Médico de Familia no espera tiempos mejores, los hace mejores Programa de la especialidad de Medicina Familiar y Comunitaria 2005

\section{Introducción}

La Medicina de Familia nace en España al amparo de tres marcos básicos: el resurgimiento del modelo biopsicosocial, la creación de un Sistema Nacional de Salud (SNS), que define la Atención Primaria (AP) como su función central, y la creación de la especialidad de Medicina Familiar y Comunitaria (MFyC). ${ }^{1}$

Las características fundamentales del SNS ${ }^{2}$ español son:

- Financiación pública mediante presupuestos generales del Estado, universalidad y gratuidad de los servicios sanitarios.

- Derechos y deberes definidos por marco legal.

- Gestión pública y provisión, que aun siendo hegemónicamente pública, utiliza también recursos privados.

- Acceso a la asistencia a través de la AP.

- Descentralización política y de gestión en las Comunidades Autónomas (CCAA).

- Organización territorial de los servicios de salud en Áreas de Salud y Zonas Básicas de Salud definidas por las CCAA en mapas sanitarios, según criterios sociodemográficos y de accesibilidad.

- Prestación de una atención integral de la salud en un marco de garantía de calidad.

- Integración de estructuras y servicios públicos en el SNS.

\section{La Atención Primaria de Salud}

De estas características del SNS, cabe destacar el acceso a la asistencia a través de la AP, que se ha constituido en un elemento clave para el desarrollo del SNS español y cuya reforma ha influido muy positivamente en la calidad de la atención sanitaria prestada a la población.

La red de centros de salud, en los que trabajan los equipos de AP, se ha extendido al conjunto del territorio. Un Equipo de AP atiende una Zona Básica de Salud, que en términos generales incluye unos 25000 habitantes y está constituido por varios médicos de familia (MF), pediatras, enfermeras, auxiliares de enfermería, administrativos, celadores, matronas, trabajadores sociales junto a equipos de apoyo (fisioterapia, salud mental). De los 114.326 médicos que trabajaban en el 
SNS ${ }^{2-4}$ en 2012: 35167 eran Médicos de AP (MF: 28743 y Pediatras: 6424) lo que representa el 30,7\% de los médicos del SNS. Esto lleva a que hay, de media, un MF por cada 1356 habitantes (en un rango entre CCAA de un MF por cada 926-1669 habitantes). Estos equipos desarrollan su labor en 3003 centros de salud y 10116 consultorios locales, a los cuales se desplazan los profesionales de los centros de salud de zona, con el fin de acercar los servicios básicos a la población que reside en núcleos dispersos. En 2011 se han producido en AP 418,4 millones de consultas (279 millones de consultas por MF y pediatras y el resto por enfermería). La frecuentación media, es decir, el número de visitas por persona y ańo, ha sido de 5,48 a MF, 4,92 a pediatría y 2,82 a enfermería. La tasa de resolución se sitúa en torno al 92\%.

Las mejoras introducidas por el modelo de $\mathrm{AP}^{5}$ se recogen en la Tabla 1 y han redundado en un mejor nivel científicotécnico, con mejoras notables en la oferta de servicios y en los niveles de salud y de satisfacción de los ciudadanos receptores de la AP. Así, en 2012, el 87,6\% de los ciudadanos consideraban como "muy buena" y "buena" la atención sanitaria recibida por el MF, el $83,4 \%$ de ellos valoraba positivamente la atención prestada por los médicos del hospital, y el $79.4 \%$ valoraba positivamente la atención ofrecida por los médicos de Urgencias. La AP, a lo largo de estos años y de forma reiterada, es el nivel sanitario mejor considerado por la población. ${ }^{6}$

Tabla 1. Mejoras introducidas por el modelo de Atención Primaria.

\begin{tabular}{|c|c|}
\hline MEJORA & IMPACTO \\
\hline $\begin{array}{l}\text { Incorporación de los especialistas en Medicina Familiar y Comunitaria, Pediatras y Enfermeras a los Equipos de } \\
\text { Atención Primaria con renovación profesional y de mejora del nivel científico-técnico. }\end{array}$ & Viabilidad competencial \\
\hline Asignación de las personas a un médico de familia. & Longitudinalidad \\
\hline $\begin{array}{l}\text { Desarrollo del enfoque biopsicosocial e integración de la promoción de salud y de prevención de enfermedades con } \\
\text { las actividades de curación, rehabilitación y cuidado paliativo. }\end{array}$ & Globalidad \\
\hline Territorialización de la Atención. & Equidad en el acceso \\
\hline Trabajo en equipo. & Multidisciplinariedad \\
\hline Trabajo en, con y para las familias y la comunidad. & Enfoque familiar y comunitario \\
\hline $\begin{array}{l}\text { Jornadas de trabajo de dedicación completa ( } 37,5 \text { horas semanales) y salarios equiparables entre los dos niveles } \\
\text { asistenciales (Atención Primaria y hospital). }\end{array}$ & Equidad interna \\
\hline $\begin{array}{l}\text { Prestación de unos servicios comunes a todos los ciudadanos garantizados por Ley e introducción de las carteras } \\
\text { de servicios. }\end{array}$ & Equidad en la prestación \\
\hline Introducción de una cultura de evaluación de la calidad de esos servicios. & Garantía de Calidad \\
\hline $\begin{array}{l}\text { Generalización del uso de la historia clínica inicialmente en papel y actualmente informatizada y conectada con la } \\
\text { historia de hospital. }\end{array}$ & Sistemas de información \\
\hline $\begin{array}{l}\text { Incorporación de la gestión de la atención como una función propia de Atención Primaria, incluyendo la gestión de } \\
\text { la actividad, la gestión clínica y la gestión de la calidad. }\end{array}$ & Sistemas de gestión propia \\
\hline $\begin{array}{l}\text { Incorporación de la formación de estudiantes de medicina y enfermería y creación de los centros de salud universitarios. } \\
\text { Incorporación de la formación especializada con la red de centros de salud docentes agrupados inicialmente en } \\
\text { Unidades Docentes de Medicina Familiar y, actualmente, con la creación de la especialidad de Enfermería Familiar } \\
\text { y Comunitaria, en las unidades docentes multiprofesionales de Atención Familiar y Comunitaria. }\end{array}$ & $\begin{array}{l}\text { Desarrollo académico con Formación universitaria } \\
\text { Formación Especializada }\end{array}$ \\
\hline Incorporación de la investigación en Atención Primaria. & Investigación \\
\hline Incremento de la capacidad de Resolución de la Atención Primaria. & Eficacia de nivel \\
\hline Mejora de los indicadores de salud imputables a la AP. & Efectividad \\
\hline Mejora de la satisfacción de los usuarios. & Viabilidad por satisfacción de la población \\
\hline
\end{tabular}

Sin embargo, aunque la valoración global de la AP en España es muy positiva por sus grandes fortalezas al ser un sistema más equitativo, más efectivo, más eficiente y viable, tiene aún importantes debilidades como son el, todavía, fuerte hospitalocentrismo y la inadecuada distribución de la financiación. El gasto sanitario público en España supuso en 2010 el 7,1\% del PIB. El gasto sanitario público en AP en 2010 se sitúa en 190,18 euros por cada habitante protegido y año. ${ }^{7}$ Sólo el 18\% de dicho gasto corresponde a AP y la decisión sobre el mismo no depende de ella. La AP, siendo clave, no es aún la función central real del sistema, su autonomía de organización y gestión es limitada, hay bolsas de ineficiencia (burocratización, múltiples sistemas de información) y su prestigio y reconocimiento no es el que debería ser. Además, la crisis económica supone una amenaza ante la posibilidad de la introducción del co-pago y la privatización. La II Conferencia de Presidentes de CCAA ya destacó, en Julio de $2005,{ }^{8}$ tanto la necesidad de impulsar la AP como el compromiso de apoyar 
su capacidad de resolución y sus recursos, valorizándola como función central. En una encuesta realizada a 5299 estudiantes de Medicina, el 90\% consideraban la Medicina de Familia como la especialidad con mayor impacto social y, sin embargo, sólo el $20 \%$ consideraba que tenía un elevado prestigio como profesión. ${ }^{9}$

Es reseńable que todos los indicadores relacionados con la presencia de AP han mejorado de forma importante. En la evaluación 2007-2012 de la Estrategia $\mathrm{AP} \mathrm{XXI}^{7}$ se enumeran los resultados en salud y en varios indicadores de actividad asistencial.

\section{El sistema de formación especializada español}

El sistema de formación especializada, a través de residencia, ha sido durante estos 35 años, indudablemente, uno de los motores que ha contribuido a elevar de forma homogénea la calidad asistencial y la seguridad de los pacientes, alcanzando altas cotas de calidad en relación a los países de nuestro entorno socio-económico.

Dicho sistema centrado en el que aprende, consiste en una práctica clínica programada y retribuida, que permite la asunción progresiva de responsabilidades y un nivel decreciente de supervisión a medida que se avanza en el proceso formativo, con una vinculación laboral entre el especialista en formación y el Servicio de Salud en el que está desarrollando el correspondiente programa formativo.

El acceso a la Formación Sanitaria Especializada ${ }^{10}$ (sistema de residencia-MIR: Médico Interno Residente) se realiza mediante una prueba selectiva convocada por el Ministerio de Sanidad, que es una evaluación teórica estatal única para todas las especialidades. Está conformada por 225 preguntas tipo test con respuestas múltiples, con 10 preguntas de reserva, que debe ser contestada en 5 horas. El examen es realizado en diferentes ciudades el mismo día. Es necesario obtener al menos una nota igual al 35\% de la media de los 10 mejores para acceder a las plazas.

La puntuación final es la suma de la puntuación obtenida en el examen ( $90 \%$ de la nota final) más la valoración del expediente académico (10\% de la misma). Se clasifica a todos los aspirantes y según esa nota, eligen especialidad y Unidad Docente en la sede del Ministerio de Sanidad en Madrid. El cupo para discapacitados es del 7\%. El cupo para extracomunitarios es del 8\%. Desde 2001 a 2012 se han formado como especialistas en España médicos no españoles procedentes de 109 países. Cabe destacar Colombia con 5.821; Perú 5.273; R. Dominicana 2.265; Venezuela 1.788; Bolivia 1.663; México 1.660; Italia 1.468; Ecuador 1.392; Argentina 1.015; Cuba 876; Rumanía 597; Honduras 399; Marruecos 383; Paraguay 361; Brasil 255.

Habitualmente el examen se convoca en Septiembre, se lleva a cabo en Enero-Febrero y los participantes eligen plazas en Abril, para incorporarse a la Formación Sanitaria Especializada en Mayo. En la última convocatoria de $2013^{11}$ se han adjudicado 6689 plazas, siendo 1775 para MFyC (26,5\%).

Las especialidades con formación mediante residencia en España son 55, de las cuales 40 son especialidades exclusivamente médicas, y el resto son especialidades a las que se accede con otras titulaciones, como biología, bioquímica, farmacia y química. Los programas formativos para médicos son de 4-5 años (Tabla 2) ${ }^{12}$ y han sido publicados en el Boletín Oficial del Estado, lo que concede un marco legal de garantías.

La formación se desarrolla en dispositivos acreditados para la formación especializada, de acuerdo a unos requisitos o estándares que incluyen, entre otros, unos mínimos relativos a recursos humanos y físicos, actividad asistencial, actividad docente e investigadora y calidad. ${ }^{13}$

\section{La especialidad de Medicina Familiar y Comunitaria (MFyC)}

El título oficial de Médico Especialista en Medicina Familiar y Comunitaria fue creado a través del Real Decreto $3303 / 1978,{ }^{14}$ de 29 de diciembre, en el marco de formación especializada mediante residencia en los centros sanitarios como sistema ordinario y habitual según el Real Decreto 2015/78. ${ }^{15}$ Se dispone que "[...] el médico de familia constituye la figura fundamental del sistema sanitario y tiene como misión realizar una atención médica integrada y completa a los miembros de la comunidad." ${ }^{4}$

Se iniciaba así, en España, una formación específica para los MF que, posteriormente, y a través de la Directiva 86/457/ $\mathrm{CEE},{ }^{16}$ del Consejo de las Comunidades Europeas, fue implantada con carácter general en todos los Estados miembros de la actual Unión Europea. Aunque el citado Real Decreto establecía las normas transitorias para el acceso al título de la 
Tabla 2. Especialidades médicas vía residencia y años de formación.

\begin{tabular}{lclc}
\hline \multicolumn{1}{c}{ Especialidad } & Años & & Especialidad \\
\hline Alergología & $\mathbf{4}$ & Medicina Familiar y Comunitaria & $\mathbf{4}$ \\
Análisis Clínicos & $\mathbf{4}$ & Medicina Física y Rehabilitación & $\mathbf{4}$ \\
Anatomía Patológica & $\mathbf{4}$ & Medicina Intensiva & $\mathbf{5}$ \\
Anestesiología y Reanimación & $\mathbf{4}$ & Medicina Interna & $\mathbf{5}$ \\
Angiología y Cirugía Vascular & $\mathbf{5}$ & Medicina Nuclear & $\mathbf{4}$ \\
Aparato Digestivo & $\mathbf{4}$ & Medicina Preventiva y Salud Pública & $\mathbf{4}$ \\
Bioquímica Clínica & $\mathbf{4}$ & Microbiología y Parasitología & $\mathbf{4}$ \\
Cardiología & $\mathbf{5}$ & Nefrología & $\mathbf{4}$ \\
Cirugía Cardiovascular & $\mathbf{5}$ & Neumología & $\mathbf{4}$ \\
Cirugía General y del Aparato Digestivo & $\mathbf{5}$ & Neurocirugía & $\mathbf{5}$ \\
Cirugía Oral y Maxilofacial & $\mathbf{5}$ & Neurofisiología Clínica & $\mathbf{4}$ \\
Cirugía Ortopédica y Traumatología & $\mathbf{5}$ & Neurología & $\mathbf{4}$ \\
Cirugía Pediátrica & $\mathbf{5}$ & Obstetricia y Ginecología & $\mathbf{4}$ \\
Cirugía Plástica, Estética y Reparadora & $\mathbf{5}$ & Oftalmología & $\mathbf{4}$ \\
Cirugía Torácica & $\mathbf{5}$ & Oncología Médica & $\mathbf{5}$ \\
Dermatología Médico-Quirúrgica y Venereología & $\mathbf{4}$ & Oncología Radioterápica & $\mathbf{4}$ \\
Endocrinología y Nutrición & $\mathbf{4}$ & Otorrinolaringología & $\mathbf{4}$ \\
Farmacología Clínica & $\mathbf{4}$ & Pediatría y sus áreas & $\mathbf{4}$ \\
Geriatría & $\mathbf{4}$ & Psiquiatría & $\mathbf{4}$ \\
Hematología y Hemoterapia & $\mathbf{4}$ & Radiodiagnóstico & $\mathbf{4}$ \\
Inmunología & $\mathbf{4}$ & Reumatología & $\mathbf{4}$ \\
Medicina del Trabajo & $\mathbf{4}$ & Urología & $\mathbf{5}$ \\
\hline
\end{tabular}

nueva especialidad por parte de los profesionales que ejercían con anterioridad a su creación, estas medidas se revelaron insuficientes a partir del 1 de enero de 1995, fecha en la que, conforme a lo previsto en la Directiva 93/16/CEE, ${ }^{17}$ entró en vigor el requisito de ostentar el título de Médico Especialista en MFyC, o la certificación a que se refiere el Real Decreto 853/1993,${ }^{16}$ de 4 de Junio, para desempeñar plazas de Médico de Familia (denominación que adopta la Medicina General con este Real Decreto), en centros o servicios sanitarios integrados en el SNS. Esta situación motivó la adopción del Real Decreto 931/1995, ${ }^{18}$ de 9 de junio, relativo al acceso a la formación como Especialistas en MFyC de los Licenciados en Medicina posteriores al 1 de Enero de 1995. En 1998 el Real Decreto 1753/199819 establece como vía de homologación de la especialidad una formación de 300 horas, 5 años de trabajo como Médico de Familia y una prueba ECOE (Evaluación Clínica Objetiva y Estructurada) de 9 estaciones. Comienza en 2002 y la última convocatoria es en 2013, con prueba prevista en el primer semestre de 2014. Hasta el momento actual han sido evaluados 9.377 candidatos, con un coste total de 3.160.996 €, financiado por el Ministerio de Sanidad, con un porcentaje de no aptos del 2,41\%. ${ }^{20}$

Con la creación de la especialidad de MFyC se desarrolla una de las estrategias claves para la consolidación de la reforma del sistema sanitario español. En todo momento se ha sustentado la formación en la evidencia de que la AP debe ser proporcionada por médicos bien formados y entrenados en su práctica y en la globalidad, longitudinalidad, abordaje familiar y comunitario, accesibilidad, siendo así más efectivos y más eficientes que otros especialistas para la prestación de las funciones propias de la AP de salud. Desde hace muchos años hay sólidas evidencias que demuestran que cuantos más MF tiene un sistema mejor son sus indicadores de salud (mortalidad por todas las causas, por cáncer, enfermedades cardio y cerebrovasculares). ${ }^{21-23}$ Recientes publicaciones objetivan claramente las grandes ventajas de la organización de los servicios de salud en torno a la AP y al Médico de Familia. Así Kringos et al. ${ }^{24}$ nos demuestra que la influencia del número de MF es tal, que incrementos de $1 \mathrm{MF} / 100.000$ habitantes se sigue de descensos de la mortalidad de 0,65/100.000.

Esto explica el desarrollo de la formación especializada en MFyC en Europa. Se ha pasado en pocos años de no exigir formación específica para ejercer la Medicina de Familia a una formación específica que era inicialmente de 2 años para pasar rápidamente a 3 ańos y situarnos en el momento actual en Europa en una horquilla de 3 a 6 años de formación (Tabla 3). ${ }^{25}$ 
Tabla 3. Formación en Medicina de Familia en países europeos.

\begin{tabular}{|c|c|c|c|c|c|c|c|c|}
\hline País & Años ${ }^{a}$ & $\mathrm{H} / \mathrm{CS}^{b}$ & Programa $^{c}$ & Rotaciones $^{d}$ & $\mathrm{~T} / \mathrm{P}^{\mathrm{e}}$ & Evaluación $^{\dagger}$ & Responsables $^{g}$ & Obligatorio \\
\hline Alemania & 5 & $=/=$ & $\mathrm{SI}$ & NO & $80 \mathrm{H}$ & $\mathrm{SI}$ & $\mathrm{G} / \mathrm{P}$ & $\mathrm{SI}$ \\
\hline Austria & 3 & $+/-$ & $\mathrm{SI}$ & $\mathrm{SI}$ & $\mathrm{OH}$ & $\mathrm{SI}$ & $G / P$ & SI \\
\hline Bélgica & 3 & $-1+$ & $\mathrm{SI}$ & NO & $300 \mathrm{H}$ & $\mathrm{SI}$ & $U / G$ & $\mathrm{SI}$ \\
\hline Bulgaria & 3 & $=/=$ & SI & $\mathrm{SI}$ & $250 \mathrm{H}$ & $\mathrm{SI}$ & $U / G$ & NO \\
\hline Croacia & 4 & $=/=$ & $\mathrm{SI}$ & NO & $400 \mathrm{H}$ & $\mathrm{SI}$ & $U / G / P$ & NO \\
\hline Dinamarca & 5 & $=/=$ & $\mathrm{SI}$ & $\mathrm{SI}$ & $320 \mathrm{H}$ & NO & G & $\mathrm{SI}$ \\
\hline Eslovaquia & 3.5 & $+/-$ & $\mathrm{SI}$ & $\mathrm{SI}$ & $100 \mathrm{H}$ & $\mathrm{SI}$ & $U$ & $\mathrm{SI}$ \\
\hline Eslovenia & 4 & $=/=$ & $\mathrm{SI}$ & $\mathrm{SI}$ & $360 \mathrm{H}$ & $\mathrm{SI}$ & $\mathrm{U} / \mathrm{P}$ & $\mathrm{SI}$ \\
\hline España & 4 & $=/=$ & $\mathrm{SI}$ & $\mathrm{SI}$ & $300 \mathrm{H}$ & $\mathrm{SI}$ & $\mathrm{G} / \mathrm{P}$ & $\mathrm{SI}$ \\
\hline Estonia & 3 & $=/=$ & SI & $\mathrm{SI}$ & $200 \mathrm{H}$ & SI & U & SI \\
\hline Finlandia & 6 & $-1+$ & $\mathrm{SI}$ & NO & $120 \mathrm{H}$ & $\mathrm{SI}$ & $U$ & $\mathrm{SI}$ \\
\hline Francia & 3 & $+/-$ & $\mathrm{SI}$ & NO & $150 \mathrm{H}$ & NO & $U$ & $\mathrm{SI}$ \\
\hline Gran Bretaña & 3 & $=/=$ & $\mathrm{SI}$ & NO & $20 \mathrm{H}$ & $\mathrm{SI}$ & $\mathrm{G} / \mathrm{P}$ & $\mathrm{SI}$ \\
\hline Grecia & 4 & $+/-$ & SI & $\mathrm{SI}$ & $80 \mathrm{H}$ & SI & G & $\mathrm{SI}$ \\
\hline Hungría & 3 & $-1+$ & SI & $\mathrm{SI}$ & $60 \mathrm{H}$ & SI & U & $\mathrm{SI}$ \\
\hline Países Bajos & 3 & $-1+$ & $\mathrm{SI}$ & $\mathrm{SI}$ & $\mathrm{OH}$ & NO & $U / G$ & $\mathrm{SI}$ \\
\hline Islandia & 5 & $=/=$ & $\mathrm{SI}$ & $\mathrm{SI}$ & $100 \mathrm{H}$ & NO & $\mathrm{U} / \mathrm{G} / \mathrm{P}$ & NO \\
\hline Irlanda & 4 & $=/=$ & SI & $\mathrm{SI}$ & $\mathrm{OH}$ & SI & G & SI \\
\hline Italia & 3 & $+/-$ & SI & NO & $400 \mathrm{H}$ & SI & G & SI \\
\hline Letonia & 3 & $=/=$ & $\mathrm{SI}$ & $\mathrm{SI}$ & SD & $\mathrm{SI}$ & $U$ & $\mathrm{SI}$ \\
\hline Malta & 3 & $=/=$ & $\mathrm{SI}$ & $\mathrm{SI}$ & $500 \mathrm{H}$ & $\mathrm{SI}$ & $\mathrm{G} / \mathrm{P}$ & $\mathrm{SI}$ \\
\hline Noruega & 5 & $-1+$ & $\mathrm{SI}$ & NO & $\mathrm{OH}$ & NO & $P$ & $\mathrm{NO}^{*}$ \\
\hline Polonia & 4 & $-1+$ & SI & $\mathrm{SI}$ & $308 \mathrm{H}$ & NO & $U / G$ & $\mathrm{SI}$ \\
\hline Portugal & 4 & $-/+$ & $\mathrm{SI}$ & $\mathrm{SI}$ & $180 \mathrm{H}$ & $\mathrm{SI}$ & G & $\mathrm{SI}$ \\
\hline República Checa & 3 & $=/=$ & $\mathrm{SI}$ & $\mathrm{SI}$ & $48 \mathrm{H}$ & $\mathrm{SI}$ & G & $\mathrm{SI}$ \\
\hline Rumanía & 3 & $=/=$ & SI & $\mathrm{SI}$ & $264 \mathrm{H}$ & SI & $\mathrm{U} / \mathrm{G} / \mathrm{P}$ & $\mathrm{SI}$ \\
\hline Suecia & 5 & $-1+$ & SI & NO & $2000 \mathrm{H}$ & NO & $G / P$ & $\mathrm{SI}$ \\
\hline Suiza & 5 & $+/-$ & $\mathrm{SI}$ & NO & $\mathrm{OH}$ & $\mathrm{SI}$ & $G / P$ & $\mathrm{SI}$ \\
\hline
\end{tabular}

Elaboración propia. Fuente Euract 2012. Wonca. ${ }^{25}$ a Número de años de formación; ' ${ }^{\circ}$ Tiempo de formación en Hospital y en Centro de Salud:50\% =/=; Más tiempo en H:+/-;

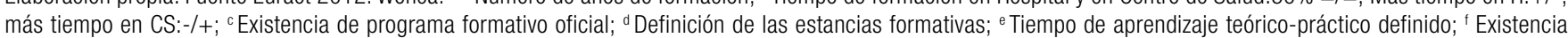
de evaluación final de residencia; ${ }^{9}$ Responsables del desarrollo de esta formación: Universidad (U), Gobierno (G), Profesión (P); ' 0 bligatorio para el ejercicio de la práctica profesional; * Mayor remuneración en los especialistas; SD: sin determinar.

\section{El programa formativo de la especialidad de Medicina Familiar y Comunitaria española}

La especialidad de MFyC es una de las 44 especialidades médicas vía residencia reconocidas en España (Tabla 2). La formación se desarrolla en las 97 Unidades Docentes (UUDD) de España, que cuenta con 887 Centros de Salud y 212 hospitales acreditados para la formación especializada en MFyC. Recientemente, con la creación de la especialidad de Enfermería Familiar y Comunitaria, las tradicionales Unidades Docentes de $\mathrm{MFyC}$ se están reacreditando como "Unidades Docentes Multiprofesionales de Atención Familiar y Comunitaria" (UDMAFyC), para englobar a ambos tipos de profesionales en formación. ${ }^{26}$

El tiempo de formación especializada abarcó, en un principio, un periodo de 3 años, hasta que se establece definitivamente en 4 años, en el año 2005, fecha en que se aprobó el tercer y último Programa Oficial de la Especialidad, que es el referente actual donde se detallan todas las competencias que debe adquirir el médico en formación (Tabla 4).

El programa actualmente vigente de la especialidad de MFyC, elaborado por la Comisión Nacional de la Especialidad (CNE) de MFyC, fue aprobado por el Ministerio de Sanidad y Consumo español el 3 de Mayo de 2005 y aparece en el Boletín Oficial del Estado (BOE no ${ }^{\text {105) }}$ mediante la Orden SCO/1198/2005.27

Se trata de un programa orientado por competencias, siendo el primer programa español de especialización médica con esta orientación. ${ }^{28} \mathrm{Ha}$ sido traducido al ruso por la OMS y al inglés..$^{29} \mathrm{El}$ proceso de elaboración de este programa se inició a finales de 1999, en el seno de la CNE de MFyC, y nace de la firme voluntad de actualizar y consolidar el cuerpo doctrinal y los valores de la MFyC y de un profundo compromiso con la formación y con el SNS. Lo avalan cinco años de trabajo en 
Tabla 4. Competencias del médico de familia: áreas docentes.

\begin{tabular}{|c|c|c|}
\hline \multicolumn{3}{|c|}{ LOS VALORES PROFESIONALES } \\
\hline \multirow{10}{*}{ ESENCIALES } & LA COMUNICACIÓN & \\
\hline & ASISTENCIAL & \\
\hline & EL RAZONAMIENTO CLÍNICO & \\
\hline & \multirow{6}{*}{ LA GESTIÓN DE LA ATENCIÓN } & La gestión clínica \\
\hline & & El trabajo en equipo \\
\hline & & La gestión y organización de la actividad \\
\hline & & Los sistemas de información \\
\hline & & La gestión de la calidad \\
\hline & & La responsabilidad civil y médico-legal \\
\hline & LA BIOÉTICA & \\
\hline \multirow{25}{*}{ ATENCIÓN AL INDIVIDUO } & \multirow{16}{*}{$\begin{array}{c}\text { ABORDAJE DE NECESIDADES Y } \\
\text { PROBLEMAS DE SALUD }\end{array}$} & Factores de riesgo y problemas cardiovasculares \\
\hline & & Problemas respiratorios \\
\hline & & Problemas del tracto digestivo y del hígado \\
\hline & & Problemas infecciosos \\
\hline & & Problemas metabólicos y endocrinológicos \\
\hline & & Problemas de la conducta y de la relación. Problemas de salud menta \\
\hline & & Problemas del sistema nervioso \\
\hline & & Problemas hematológicos \\
\hline & & Problemas de la piel \\
\hline & & Traumatismos, accidentes e intoxicaciones \\
\hline & & Problemas de la función renal y de las vías urinarias \\
\hline & & Problemas músculo-esqueléticos \\
\hline & & Problemas de cara-nariz-boca-garganta y oídos \\
\hline & & Problemas de los ojos \\
\hline & & Conductas de riesgo \\
\hline & & Atención a las urgencias y emergencias \\
\hline & \multirow{9}{*}{$\begin{array}{c}\text { ABORDAJE DE GRUPOS } \\
\text { POBLACIONALES Y GRUPOS } \\
\text { CON FACTORES DE RIESGO }\end{array}$} & Atención al niño \\
\hline & & Atención al adolescente \\
\hline & & Atención a la mujer \\
\hline & & Atención al adulto \\
\hline & & Atención a los trabajadores \\
\hline & & Atención al anciano \\
\hline & & Atención al paciente inmovilizado \\
\hline & & Atención al paciente terminal, al duelo y a la cuidadora \\
\hline & & Atención a las situaciones de riesgo familiar y social \\
\hline \multicolumn{3}{|l|}{ ATENCIÓN A LA FAMILIA } \\
\hline \multicolumn{3}{|l|}{ ATENCIÓN A LA COMUNIDAD } \\
\hline FORMACIÓN, DOCENCIA E INI & ГIGACIÓN & \\
\hline
\end{tabular}

sus diferentes fases y la colaboración de la estructura docente y la semFYC, coordinados por la CNE con más de $200 \mathrm{MF}$ elaborando el proyecto.

Este programa, largamente trabajado, se define ${ }^{28}$ por:

- La incorporación en la formación de los valores profesionales del médico de familia.

- La revisión del perfil profesional.

- La incorporación de competencias esenciales: la comunicación, el razonamiento clínico, la gestión de la atención y la bioética.

- La adaptación a las necesidades sociales y sanitarias, que se verían reflejadas en el área de atención al individuo, tanto en su bloque de atención a problemas y necesidades de salud como en el área de abordaje de grupos poblacionales y de riesgo. 
- El cambio de terminología empleada al referirse a las áreas de atención al individuo, ya que el médico de familia no aprende cardiología, neurología o urología, sino que aprende el abordaje de problemas cardiovasculares, problemas del sistema nervioso o problemas de las vías urinarias, etc. Porque este abordaje forma parte tanto de nuestra especialidad como de las otras especialidades anteriormente mencionadas.

- La profundización en las competencias que se consideran "específicas" del médico de familia, que son la Atención a la Familia y la Atención a la Comunidad.

- El desarrollo de las competencias relativas a la formación, docencia e investigación.

\section{Características del programa}

1. La flexibilidad en el desarrollo del programa. Lo importante son las competencias determinadas por el perfil profesional: "tiempos y métodos deben estar a su servicio".

2. El Programa debe permitir la pluripotencialidad, es decir, formar MF que puedan trabajar no sólo en el Centro de salud, sino en otros entornos laborales, como las urgencias, los cuidados paliativos, la atención a grupos de riesgo, etc. (Tabla 5).

Tabla 5. Ámbitos profesionales del médico de familia.

\begin{tabular}{|c|c|}
\hline \multirow[t]{11}{*}{ Asistenciales } & Medicina de Familia en Atención Primaria. \\
\hline & Emergencias. Urgencias hospitalarias. Urgencias extrahospitalarias/puntos de atención continuada. \\
\hline & Pediatría en Atención Primaria. \\
\hline & Unidades de Atención/hospitalización a domicilio. \\
\hline & Unidades de atención a adolescente. \\
\hline & Residencias geriátricas. \\
\hline & Unidades de Cuidados Paliativos. \\
\hline & Mutuas de accidentes laborales. \\
\hline & Unidades de Valoración del daño corporal. \\
\hline & Unidades de Atención a las personas en riesgo social: inmigrantes, víctimas de violencia familiar, discapacitados... \\
\hline & Centros de Reconocimientos médicos. \\
\hline En gestión & Cargos directivos y puestos técnicos en empresas relacionadas con la sanidad, en el ámbito privado y público. \\
\hline sanitaria & Comercial de productos médicos. \\
\hline \multirow[t]{5}{*}{ En docencia } & Profesores asociados y titulares de Facultades de Medicina. \\
\hline & Profesores asociados, titulares y catedráticos de Escuelas universitarias de Enfermería. \\
\hline & Profesores de otras escuelas universitarias. \\
\hline & Formación sanitaria especializada: jefes de estudio y técnicos de Salud de UUDD de MFyC. \\
\hline & $\begin{array}{l}\text { Compaginada con la asistencia, la docencia especializada (tutor principal, tutor de apoyo colaborador docente) y la formación continuada } \\
\text { en el centro. }\end{array}$ \\
\hline Investigación & La mayor parte de la investigación médica en España se realiza en las universidades y en redes de investigación de AP. El número de \\
\hline & médicos que viven exclusivamente de la investigación es muy escaso, y la mayoría lo hacen combinando investigación y docencia. \\
\hline \multirow[t]{11}{*}{ Otras opciones laborales } & Con una preparación u oposición específica para cada una de ellas: \\
\hline & Sanidad Militar. \\
\hline & Sanidad Penitenciaria. \\
\hline & Sanidad Nacional. \\
\hline & Médico de Ayuntamiento. \\
\hline & Inspector Médico de la RENFE. \\
\hline & Inspector Médico de la Seguridad Social. \\
\hline & Médico de Registro Civil. \\
\hline & Médico de Sanidad Penitenciaria. \\
\hline & Profesor de Tecnología y Práctica Sanitaria (Formación Profesional). \\
\hline & Reespecialización: el 15\% de los médicos de familia hacen otra especialidad (la media en otras especialidades oscila entre 4-60\%). \\
\hline Trabajo en el extranjero & Suécia, Portugal, Reino Unido, Irlanda. \\
\hline
\end{tabular}


3. El Programa debe pivotar en el eje tutor-residente. La formación central se da en el centro de salud, pero se deben proporcionar estancias electivas y se deben ampliar los entornos docentes con el objetivo de adquirir las competencias. El Tutor principal es el responsable de la formación tutorizada, continuada y estructurada, independientemente de las estancias formativas en el Centro de Salud. Se determina un contacto amplio inicial con AP (3-6 meses) y que al menos el 50\% del tiempo de la residencia se efectúe en el Centro de Salud, es decir, que de los 44 meses de formación al menos 22 sean en MF en AP, con un tiempo de estancia formativa en Centro de Salud todos los años. La formación debe ser individualizada, con un incremento progresivo de la responsabilidad.

4. Se establece el formato estructural del Programa. Se definen las cinco áreas competenciales (Tabla 4) y por cada área se determinan los objetivos específicos y las actividades propias para la adquisición de estas competencias, incluyendo conocimientos, habilidades y actitudes. Para desarrollar estos aspectos se confecciona una ficha que recoge: área competencial, objetivos específicos, actividades docentes propias agrupadas según prioridad y nivel de responsabilidad, recomendaciones sobre la metodología docente, el lugar de aprendizaje (Centro de Salud, estancia en un servicio de la Unidad Docente, rotación externa, etc.), el tiempo de aprendizaje, la evaluación y las lecturas recomendadas.

La evaluación formativa cobra especial relevancia en el marco de una formación tutorizada y personalizada. Y esa es la razón de la elaboración y puesta en marcha de un nuevo Libro del Especialista en Formación (LEF). ${ }^{30,31}$

La organización pivota básicamente en las UUDD, que no sólo deben organizar las estancias formativas, sino definir las actividades y los métodos que en su entorno son los más operativos para garantizar la adquisición de competencias.

El grado de implicación de los Tutores en el desarrollo del Programa es piedra angular. Su formación en los contenidos propios de la especialidad, en metodología docente y en evaluación formativa son determinantes. El apoyo a la función de Tutores por parte de la Administración es fundamental y se precisan actividades formativas, adaptación de las condiciones estructurales a la docencia y puesta en marcha de actuaciones que incentiven a los Tutores. Por ello se determinan requisitos estructurales de idoneidad de presión asistencial, tiempo de dedicación a la asistencia con tiempo específico para la docencia y tiempos mínimos por consulta, que son requisitos que hacen "saludable" el entorno para el aprendizaje.

\section{Líneas de desarrollo}

La especialización tiene innegables ventajas, ya que mejora la calidad de la asistencia y favorece el avance en el conocimiento mediante la investigación y la docencia. Sin embargo, la excesiva superespecialización también tiene algunos inconvenientes al provocar la atomización de la asistencia a un mismo paciente e incluso la desconexión entre los distintos profesionales con distintas áreas de interés. Con el agravante de que cuanto más se segmente el mercado, mayores le parecerán las necesidades al planificador. ${ }^{32}$ Por otra parte, los mismos avances de la ciencia médica introducen una cierta transversalidad entre las especialidades clásicas y ello configura un nuevo mapa de competencias. En muchos dispositivos asistenciales se están experimentando nuevas formas de organización con la integración de distintos especialistas que ponen en común conocimientos y habilidades para gestionar casos y resolver demandas de forma más eficiente.

Por ello, hemos asistido a un intenso debate sobre cómo hallar un equilibrio entre la necesaria superespecialización y la necesidad de integrar la mayoría de los aspectos de la práctica médica prevalente en el núcleo central de la formación de todos los especialistas. ${ }^{33-35}$ Tener una buena base de conocimientos troncales aumentará la eficacia y la calidad de las prestaciones sanitarias y evitará derivaciones y consultas innecesarias, favorecerá la colaboración entre servicios y entre niveles asistenciales, que acarreará menos molestias al paciente, evitará la polifarmacia injustificada y favorecerá la eficiencia del sistema sanitario.

El proyecto de desarrollo de la troncalidad ${ }^{36}$ enmarcado en la Ley de Ordenación de Profesiones Sanitarias del 2003, ${ }^{37}$ actualmente pendiente de aprobación, define tres espacios formativos en la formación especializada: la formación especializada troncal, la formación especializada específica y las áreas de capacitación específica (o alta especialización). Se considera la troncalidad en formación especializada como "[...] el conjunto de competencias comunes a varias especialidades médicas que permite la agrupación de éstas y de su proceso formativo durante un tiempo determinado." Dicho proyecto determina la existencia de 5 troncos: el tronco médico, el tronco quirúrgico, el tronco de imagen, el tronco de laboratorio y el tronco de psiquiatría. Las especialidades que formen parte de un mismo tronco deben tener competencias comunes, beneficiarse de estancias formativas comunes y deben compartir áreas en las que el trabajo conjunto mejore la efectividad y eficiencia y la satisfacción del paciente. 
Las competencias genéricas son comunes a todos $\operatorname{los} \operatorname{tron} \cos ^{38} \mathrm{y}$ las competencias del tronco médico tienen íntima relación con las competencias de las especialidades más pluripotenciales, como son la MFyC y la medicina interna. En definitiva, cuando se desarrolle el proyecto, en un centro de salud docente, rotarán los especialistas en formación de las especialidades de MFyC y Enfermería Familiar y Comunitaria, especialistas del tronco médico en su periodo de formación troncal y allí se seguirán desarrollando las estancias formativas de otros especialistas en formación, no incluidos en tronco médico (cirujanos, traumatólogos, dermatólogos, rehabilitadores, ginecólogos...en formación) regulados por Resolución. ${ }^{39}$

Todo esto se debe a que la Medicina de Familia y la AP son una especialidad y un ámbito sanitario de extraordinaria envergadura para la formación troncal y ésta debe iniciarse indefectiblemente en el grado. ${ }^{40}$

Por todo ello, y para concluir, es clave que, dadas las evidencias científicas, los Ministerios y organismos responsables del sistema sanitario y del sistema educativo de los países deban considerar, como objetivo claro y fundamental, el hecho de "valorizar" con financiación adecuada y empowerment a la AP, como función central, ${ }^{41}$ y a la MFyC, como disciplina, especialidad y profesión sanitaria. Porque, como diría la profesora Barbara Starfield: "[...] la Medicina de Familia debe dar forma a la reforma y no al revés." 42

\section{Referencias}

1. Casado V, Planes A, Thomas V, Vázquez JR. La Medicina de Familia y Comunitaria. Valores profesionales y perfil profesional del médico de familia. En Casado V et al (editors). Tratado de Medicina de Familia y Comunitaria. Barcelona:semFYC: EditorialMedicaPanamericama; 2012. p. 3-29.

2. Ministerio de Sanidad, Servicios Sociales e Igualdad (ES). Sistema Nacional de Salud. España 2012 [Internet]. Madrid: Ministerio de Sanidad, Servicios Sociales e Igualdad (ES); 2012. [última consulta el 2013 Dec27]. Disponible en: http://www.msssi.gob.es/organizacion/sns/docs/ sns2012/SNS012_Espanol.pdf

3. Actividad asistencial en Atención Primaria. Informe estadístico. Sistema de Información de Atención Primaria (SIAP). Año 2012 [Internet]: Madrid: Ministerio de Sanidad, Servicios Sociales e Igualdad (ES).[última consulta el 2013 Dec 27]. Disponible en: http://www.msssi.gob.es/ estadEstudios/estadisticas/estadisticas/estMinisterio/siap.htm

4. Portal Estadístico del Ministerio de Sanidad, Servicios Sociales e Igualdad. Consulta interactiva del SNS[Internet]. Madrid: Ministerio de Sanidad, Servicios Sociales e Igualdad (ES).[última consulta el 2013 Dec 27]. Disponible en:http://pestadistico.inteligenciadegestion.msssi.es/

5. Casado V, Aguilera L. La Atención Primaria de Salud. Otros ámbitos de actuación de la Medicina Familiar y Comunitaria. En Casado V et al (editors). Tratado de Medicina de Familia y Comunitaria. Barcelona:semFYC: EditorialMedicaPanamericama; 2012. p. 33-50.

6. Centro de Investigaciones Sociológicas. Barometro Sanitario 2012 [Internet]. Madrid: Ministerio de Sanidad, Servicios Sociales e lgualdad (ES); 2012 Mar-Nov [última consulta el 2013 Dec 27]. Disponible en:http://www.msssi.gob.es/estadEstudios/estadisticas/docs/BS_2012/B.S.2012total. mar.pdf

7. Ministerio de Sanidad, Servicios Sociales e Igualdad (ES). Estrategias en Atención Primaria para el siglo XXI. Proyecto AP21.Marco estratégico para la mejora de la Atención Primaria en España 2007-2012. Informe de seguimiento [Internet]. Madrid: [publisherunknown];2012 [última consulta el 2013 Dec 27]. Disponible en:http://www.msssi.gob.es/estadEstudios/estadisticas/docs/AP21_INFORME_2012.pdf

8. Ministerio de Economía y Hacienda; Ministerio de Sanidad y Consumo; Grupo de trabajo de la Conferencia de Presidentes para el análisis del gasto sanitario presidido por la IGAE.Informe para el Análisis del Gasto Sanitario [Internet].Madrid: Instituto de Estudios Fiscales;2005 Jul. 350 p. [última consulta el 2013 Dec 27]. Disponible en:http://www.ief.es/documentos/recursos/publicaciones/libros/Libros_blancos/GastoSanitario.pdf

9. Martin-Zurro A, Jimenez J, Monreal A, Mundet X, Otero A, Alonso-Coello Pet al.Medical student attitudes towards family medicine in Spain: a statewide analysis.BMC FamilyPractice [Internet]. 2012 [última consulta el 2013 Dec 27];13:47. Disponible en: http://www.biomedcentral. com/1471-2296/13/47

10. Ministerio de Sanidad, Servicios Sociales e Igualdad (ES). El acceso a la plazas de formación [Internet]: Madrid; 2013 [última consulta el 2013 Dec 27]. Disponible en: http://sis.msssi.es/fse/PaginasDinamicas/General/PagGeneral.aspx?Menuld=QE-00\&SubMenuld=QE-02\&cDocum=1

11. Boletin Oficial del Estado. Madrid: Agencia Estatal Boletín Oficial del Estado. n.229, Sección II B.2012Sep 22.p. 672115. Disponible en: http:// www.boe.es/boe/dias/2012/09/22/pdfs/BOE-A-2012-11914.pdf

12. Ministerio de Sanidad, Servicios Sociales e Igualdad (ES). Programas Formativos de Especialidades en Ciencias de la Salud [Internet]. 2005. Disponible en:http://www.msssi.gob.es/profesionales/formacion/guiaFormacion.htm

13. Ministerio de Sanidad, Servicios Sociales e Igualdad (ES). Acreditación de centros, unidades y dispositivos docentes para la Formación Sanitaria Especializada [Internet]. Madrid; [update 2012Sep; última consulta el 2014 Mar 01]. Disponible en: http://www.msssi.gob.es/profesionales/ formacion/AcreDocCntUniForSanEsp.htm

14. Real Decreto 3303/1978, de 29 de diciembre, de regulación de la medicina de familia y comunitaria como especialidad de la profesión médica Boletin Oficial del Estado. Madrid:Agencia Estatal Boletín Oficial del Estado. n. 29. 1979 Fev 2. p. 2735-6. [última consulta el 2013 Dec 27]. Disponible en: http://www.boe.es/boe/dias/1979/02/02/pdfs/A02735-02736.pdf

15. Real Decreto 2015/1978, de 15 de julio, por el que se regula la obtención de títulos de especialidades médicas. Boletin Oficial del Estado. Madrid:Agencia Estatal Boletín Oficial del Estado. n.206. 1978Aug 29.p. 20172-4. [última consulta el 2013 Dec 27]. Disponible en: http://www. boe.es/boe/dias/1978/08/29/pdfs/A20172-20174.pdf

16. Real Decreto 853/1993, de 4 de julio, sobre ejercicio de las funciones de Médico de Medicina General en el Sistema Nacional de Salud.Boletín Oficial del Estado. Madrid:Agencia Estatal Boletín Oficial del Estado. n. 156, 1993 Jul 01. p. 20049-51. [última consulta el 2013 Dec 27]. Disponible en: http://www.boe.es/boe/dias/1993/07/01/pdfs/A20049-20051.pdf 
17. Directiva 93/16/CEE. Diario Oficial n L 165 de 07/07/1993; 0001 -0024.Disponible en: http://europa.eu/legislation_summaries/other/l23021_ es.htm

18. Real Decreto 931/1995.Boletin Oficial del Estado. Madrid: Agencia Estatal Boletín Oficial del Estado. n. 143.1995 Jun 16.P. 18065-66.[última consulta el 2013 Dec 27]. Disponible en: https://www.boe.es/boe/dias/1995/06/16/pdfs/A18065-18066.pdf

19. Boletin Oficial del Estado. Madrid: Agencia Estatal Boletín Oficial del Estado. n. 205. 1998Aug 27[última consulta 2014 Mar 01]. p. $29232-35$. Disponible en: http://www.msssi.gob.es/profesionales/formacion/docs/RD_MFYC.pdf

20. Casado V, Basora J. Assessment of Clinical Skills for the Quality of Specialists in Family and Community Medicine (track discussion).Ann Fam Med. 2013;11(6):185-7. [Última consulta el 2013Dec 27].Disponible en http://www.annfammed.org/content/11/2/185/reply

21. Greco PJ, Eisenberg JM. Changing physicians. N. Engl J Med. 1993;329(17):1271-3.

22. Epstein RM, Hundert EM. Defining and assessing professional competent. JAMA. 2002;287:226-234.

23. Grimshaw JM, Winkens RA, Shirran L, Cunningham C, Mayhew A, Thomas R et al. Interventions to improve outpatient referrals from primary care to secondary care. Cochrane Database Syst Rev.2005 Jul 20[última consulta el 2013 Dec 27];(3):CD005471. Disponible en http://www.ncbi.nlm. nih.gov/pubmed/16034981

24. Kringos DS, Boerma W, Hutchinson A, Van deer Zee J, Groenewegen P. The breadth of primary care: a systematic literature review of its core dimensions. BMC HealthServicesResearch.2010[última consulta el 2013 Dec 27];10:65.Disponible en: http://dx.doi.org/10.1186/1472-696310-65

25. Specialist Training in General Practice/Family Medicine - Dynamic Interactive Database [Internet].European Academy of teachers in General Practice/Family Medicine.c2014.Disponible en:http://www.euract.eu/resources/specialist-training

26. Boletín Oficial del Estado. Madrid: Agencia Estatal Boletín Oficial del Estado. n. 119, Sección I.2013May 18.p. 36863-73. Disponible en:http:// www.boe.es/boe/dias/2013/05/18/pdfs/BOE-A-2013-5218.pdf

27. Boletín Oficial del Estado. Madrid:Agencia Estatal Boletín Oficial del Estado. no. 105. 2005May 13. p. 15190-225. Disponible en: http://www. msssi.gob.es/profesionales/formacion/docs/mediFamiliar07.pdf

28. Casado V. La Residencia en Medicina Familiar y Comunitaria: 4 Años, Suma y Sigue. Atención Primaria. 2005.35 (extraordinario I) 5-38.

29. Sociedad Española de Medicina Familiar y Comunitaria (semFYC).Casado V, et al. Family and Comunity MedicineTraining Program, Barcelona: semFYC; 2006. Disponible en:http://www.semfyc.es/pfw_files/cma/Informacion/modulo/documentos/programa-especialidad-ingles.pdf

30. Comisión Nacional de la Especialidad de Medicina Familiar y Comunitaria. Programa Formativo de la Especialidad de Medicina Familiar y Comunitaria. Ministerio de Sanidad y Consumo. [última consulta el 2013 Dec 27]. Disponible en: http://www.msc.es/profesionales/formacion/ docs/medifamiliar.pdf

31. Ministerio de Sanidad, Servicios Sociales e Igualdad (ES). Ley 44/2003 de 21 de Noviembre, de Ordenación de las Profesiones Sanitarias, Art.20. Libro del Residente de Medicina Familiar y Comunitaria. Disponible en: http://www.msssi.gob.es/profesionales/formacion/docs/ libroResidenteMedFamiliar.pdf

32. Gonzalez B, Barber P. Oferta y Necesidad de Médicos especialistas en España (2006-2030). Palmas: Universidad de las Palmas de Gran Canaria; 2007[última consulta el 2013 Dec 27]. Disponible en http://www.msc.es/novedades/docs/necesidadesEspeciales06_30.pdf

33. Terés J, Capdevilla JA, Nonell F, Cardellach F, Bundó M y Torres M. Formación troncal de las especialidades médicas: un reto del presente para una mejora del sistema sanitario. Med Clin (Barc). 2006;127(4):139-44.

34. World Federation for Medical Education (WFME). Trilogía de la WFME. Estándares globales en Educación médica. Pregrado, postgrado y desarrollo profesional continuo. Educación médica. 2004;7(2).

35. Casado V. Troncalidad y medicina de familia. Aten primaria. 2009;41(4):179-80.

36. Ministerio de Sanidad, Servicios Sociales e Igualdad (ES). Proyecto de Real Decreto por el que se regula la troncalidad y otros aspectos del sistema de formación sanitaria especializada en ciencias de la salud. [última consulta el 2013 Dec 27]. Disponible en: http://www.msssi.gob.es/ normativa/docs/Rdecretotroncalidad.pdf

37. Ministerio de Sanidad, Servicios Sociales e Igualdad (ES).Ley 44/2003, de 21 de noviembre, de Ordenación de las Profesiones Sanitarias. BOE n.280. p. 41442-58. [última consulta el 2013 Dec 27]. Disponible en: http://www.boe.es/boe/dias/2003/11/22/pdfs/A41442-41458.pdf

38. Grupo de troncalidad del Consejo Nacional de Especialidades en Ciencias de la salud. La Troncalidad en las Especialidades Médicas. Madrid:Ministerio de Sanidad y Consumo yMinisterio de Ciencia e Innovación. Consejo Nacional de Especialidades en Ciencias de la salud; 2008. No publicado. [última consulta el 2013 Dec 27]. Disponible en http://www.sect.es/datos/WEB/PDF/Educaci\%C3\%B3n_Pregrado/La\%20 troncalidad\%20en\%20las\%20especialidades\%20m\%C3\%A9dicas\%202008.pdf

39. Resolución de la Dirección General de Recursos Humanos y servicios económicos-presupuestarios, del Ministerio de Sanidad y Consumo sobre la incorporación progresiva de una rotación en atención primaria de salud, en los programas formativos de determinadas especialidades sanitarias. 2006 Jun 15. Disponible en:http://www.areasaludplasencia.es/wasp/pdfs/4/docs/41615.pdf

40. Casado V, Bonal P, Cucalón JM, Serrano E, Suárez F. La medicina familiar y comunitaria y la universidad. Informe SESPAS 2012.Gac Sanit. 2012;26(S):69-75.

41. Ministerio de Sanidad, Servicios Sociales e Igualdad (ES).Ley 16/2003 de 28 de mayo, de Cohesión y Calidad del Sistema Nacional de Salud. BOE n’. 128. 2003May 29. p. 20567-88. [última consulta el 2013 Dec 27]. Disponible en:https://www.boe.es/diario_boe/txt. php?id=BOE-A-2003-10715

42. Starfield B. Family Medicine Should Shape Reform, Not Vice Versa. FamilyPractice Management. 2009;16(4):6. 